\title{
Enhanced Intelligent Automated Method to MSRCR Algorithm for Image Enhancement
}

\author{
Immanuel K., Dinesh Kumar A., Praveen Kumar E.
}

\begin{abstract}
The images captured by a camera may need tweaking based on the lighting situations present at the scene captured. The extremities in the lighting and the position of the object may sometime degrade the image captured. To have a right image all the time this concept was developed. This paper focuses on the automated procedure which rides over the Multi Scale Retinex with Colour Restoration Algorithm for best possible results. This is a completely automated approach where the user need not do anything apart from giving the image as the input. The main problems in photography are overcome to a great extent using this procedure.
\end{abstract}

Keywords: MSRCR, High Key, Low Key, Histogram Equalisation.

\section{INTRODUCTION}

The dynamic range of the image captured using the camera is always less than that of the human eye. To have an image in par with human vision system the Multi Scale Retinex Procedure was developed. In this procedure the captured image is multi sampled and the minor details that remain invisible are made visible. Thus overall enhancement of the image is done. There are many other techniques available for image enhancement but unlike the MSRCR model the fullness of enhancement and the fineness of details are not achieved in an automated approach. The main purpose of having image enhancement is to improve the image and make it more attractive to the perceiver than its original on which all the available image enhancement techniques target on. Also this paper focuses on how the human eyes would have perceived a scene and what needs to be done to the captured image so that it looks like the scene perceived by the human eye. The advantages of this algorithm were greatly explored and the result was tweaking the algorithm and developing this automated procedure.

Revised Manuscript Received on February 05, 2020.

* Correspondence Author

Immanuel K., Assistant Professor, Department of ECE, St. Joseph's Institute of Technology, Chennai, India. E-mail: immanuelk@live.com

Dinesh Kumar A., Assistant Professor, Department of ECE, St. Joseph's Institute of Technology, Chennai, India. E-mail: dinesheceirtt@gmail.com

Praveen Kumar E., Assistant Professor, Department of ECE, St. Joseph's Institute of Technology, Chennai, India. E-mail: epraveenkumar10@gmail.com

(C) The Authors. Published by Blue Eyes Intelligence Engineering and Sciences Publication (BEIESP). This is an open access article under the CC BY-NC-ND license (http://creativecommons.org/licenses/by-nc-nd/4.0/)

\section{MULTI SCALE RETINEX WITH COLOUR RESTORATION}

To improve the colour gamut and have wider colour rendition in images this algorithm was developed. The colour constancy of the image is also preserved. The algorithm for colour restoration is defined in the following. The MSRCR algorithm does the multi sampling of the image along with restoration of colour by considering multiband colour restoration function [1] during the enhancement procedure. Through this operation the adding up of colour levels or the subtraction of colour levels is possible giving the image a very realistic human eye view.

\section{COMPARISON OF MSRCR WITH OTHER TECHNIQUES}

In order to find out the advantages of MSRCR over other available techniques the comparison of enhanced images where done. Other techniques rely fully on the image being enhanced. Enhancement of images in other techniques is mainly the feature of dynamic range compression which results in clipping of low key and high key details. In histogram equalisation [2] the perceived scene's natural view is totally lost giving a pseudo look to the scene, so is the case with Adaptive Histogram Equalisation [3] too. With methods like white patch[4] and modified white patch too the complete enhancement is not possible. Since MSRCR algorithm is developed having the colour constancy theory in mind the results are far more superior to other algorithms.

\section{WHAT MAKES MSRCR ALGORITHM A UNIQUE CHOICE FOR THIS AUTOMATION PROJECT}

The entire MSRCR algorithm [1] can be controlled by controlling the three parameters gain, alpha \& beta. Also for additional control the c values for the SSR algorithm [1] is fixed with precision. All the other available algorithms do enhance the image but none of them increase the dynamic range of the image. So without any confusion this algorithm was selected for the project. For automation purpose few other additional parameters were added for precise control.

\section{TYPES OF IMAGES AND THEIR CORRECTIVE PROCEDURE}

An image shot can be degraded due to two factors either because of over exposure or under exposure. These two factors take place when the camera either opens the aperture more than the required value or less than the required value allowing wrong amount of light to fall on the imaging sensor. 
Even though the aperture level is controlled by strong and powerful algorithms on many cases the image gets degraded. The effective light range capable by the sensor to handle in a particular aperture also may not be wide thus resulting in loss of details in the high key areas (highlights)[2] and hardly details available in low key areas (shadows)[2]. The perfect balance of the highlights and the shadows gives a good image. The automation mainly targets on details in both shadows and highlights.

Types of Degraded Images

ii) Over Bright Images

iii) Images with correct exposure level but with reduced dynamic range

In dark images the luminance levels have to be amplified without the appearance of visible noise. In over bright images the luminance levels have to be reduced so that the hidden details will become visible. For the third category no amplification or attenuation of image levels is necessary but improvement in details is needed. For handling these three categories the original algorithm cannot be used rather some more additional control parameters attributes are necessary to realize the proposed system.

\section{MSRCR'S COMPATIBILITY FOR DARK AND BRIGHT IMAGES}

As mentioned earlier there are both dark and bright images. In an automation process the process must be capable of handling both types of images. But unfortunately the MSRCR algorithm was designed only for the dark image enhancement and the dynamic range extraction of the image. So in this project some corrections are done to the base algorithm, making it even suitable for reduction of intensity levels for very bright images. The enhancement of high key images involve the continuous subtraction of re-sampled intensity levels in the MSR stage where a surround function parameter was fixed as 25,150,250 for best results. When the first stage is subtracted from the other two stages the finer details or the smaller intensity variations are revealed from the image.

\section{AUTOMATION PROCEDURE}

An entirely new automation procedure is developed for the execution of the MSRCR algorithm on the images. The method is Adaptive Method with Contrast Control. The Steps followed are

i) The upper and lower mean intensity values for different bands are extracted from the image.

ii) Based on the extracted values the mean value is calculated and varied to suit the image.

iii) The pre-set values of alpha, beta and gain are increased or decreased based on the mean values obtained

iv) Contrast control is done in order to avoid the burning up off high key details in enhancement procedure where an additional weight factor is multiplied with the MSRCR complex result obtained after processing

$$
\begin{gathered}
R_{M S R C R i}(x, y)=G\left[W * C_{i}(x, y) R_{M S R i}(x, y)\right. \\
\pm b]
\end{gathered}
$$

i) Dark Images

The weight factor $\mathrm{W}$ is decided based on the analysis of the image by the algorithm. The value varies between 0.42 to 0.63 for best results.

For High Key Images the Process is done in the inverse manner i.e. subtraction is done.

\section{Base Processing}

$\operatorname{Imax}=\max \left(\operatorname{Imx}_{R}, \operatorname{Im} x_{B}, \operatorname{Imx} x_{G}\right)$

$\operatorname{Imin}=\min \left(\operatorname{Imn}_{\mathrm{R}}, \operatorname{Imn}_{\mathrm{B}}, \operatorname{Imn}_{\mathrm{G}}\right)$

where $\operatorname{Imx}$ is the mean max intensity of the individual bands

$$
\begin{aligned}
C_{\text {alpha }}= & \frac{(\text { Band } * \text { Normalisation Factor } 1)+(\text { Imax }- \text { UMIV })}{(\text { Imax }+ \text { Imin })} \\
C_{\text {beta }} & =\frac{(\text { Band } * \text { Normalisation Factor } 2)+(\text { Imin }- \text { LMIV })}{(\text { Imax }+ \text { Imin })}
\end{aligned}
$$

DP - Decision Parameter that decides whether subtractive or additive processing has to be preformed

$$
C_{\text {gain }}=\frac{\left(C_{\text {alpha }} * N F 1\right)+\left(C_{\text {beta }} * N F 2\right)}{U M I V+L M I V}
$$

$$
\begin{aligned}
& \text { Alpha }=\text { Default Value } \pm C_{\text {alpha }} \\
& \text { Beta }=\text { Default Value } \pm C_{\text {beta }} \\
& \text { Gain }=\text { Default Value } \pm C_{\text {gain }}
\end{aligned}
$$

UMIV - Upper mean Intensity Value

LMIV - Lower mean Intensity Value

Flowchart for the automated procedure

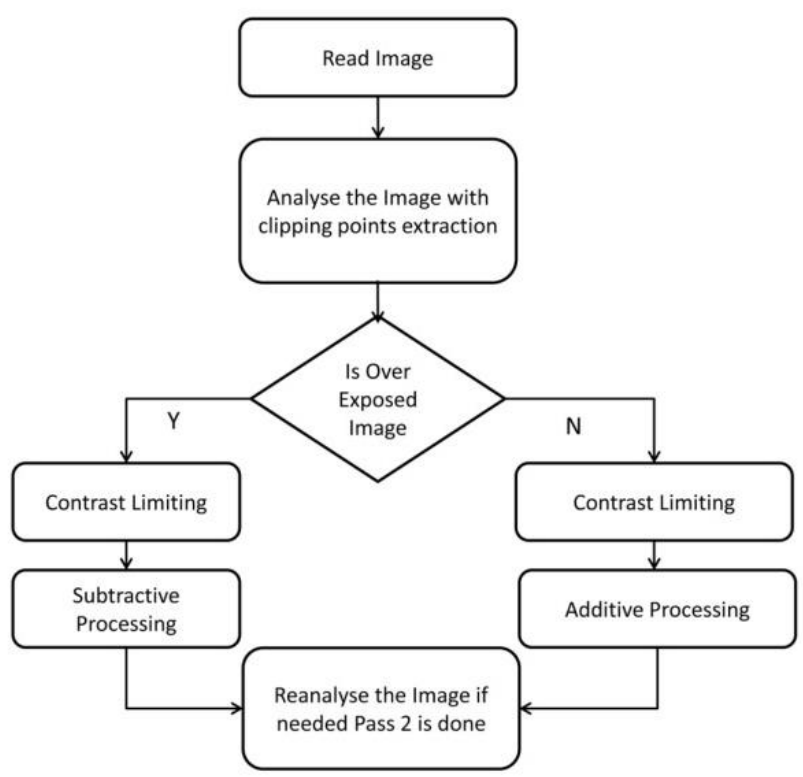

Fig 1. Flowchart for the Automation Procedure

The parameter Alpha operates on the overall intensity values of the image. Based on the extracted LMIV and UMIV the control of Alpha is done. The default value of Alpha after working with various images was fixed to be 35 for low keys images and $(50+0.5 *($ LMIV) $)$ for high key images. The Beta is the control parameter for black levels or the shadow levels of the image.

Published By: 
For a low key image the Beta value turns out to be positive since low intensity details have to be enhanced. For high key images the Beta value turns out to be negative since the overall intensity of the image has to be reduced. For certain special cases where light flares and other external entities degrade the black level of the image the Beta value turns negative so as to give a proper fix to the black level.

The default value of Beta after working with various images was fixed to be 20 for low key images and $(20+$ $0.4 *$ LMIV) for high key images. Gain parameter is indirectly linked to the Gamma curve of the image. For irregularities in the luma values this factor is varied. Small variations in the gain parameter show drastic differences in the image. For highly under exposed images gain parameter is varied a little more, as required for the image. Default value of the gain is 1 and the variations result between a plus or minus of 0.15 .

Apart from the above shown procedure to finalize the results some final enhancements like colour level fixing, normalization of intensity values within the specified range and compression of the dynamic range are also done.

\section{RESULTS}

The results contain the sample images on which the above shown procedure was applied and noted.

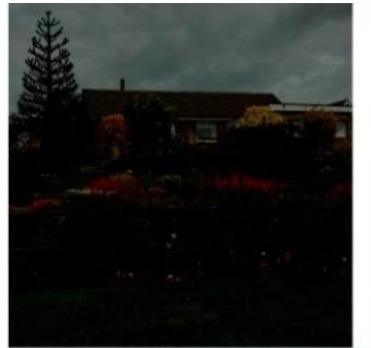

Original

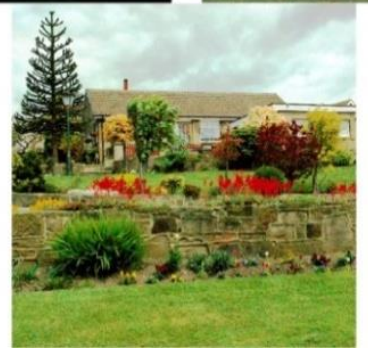

EIAMSRCRIE Output

istogram

Equalisation

Fig 2. Sample Image 1 (Low Key)and the Enhanced Image

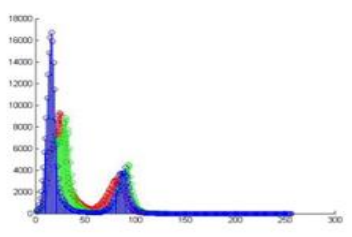

Original

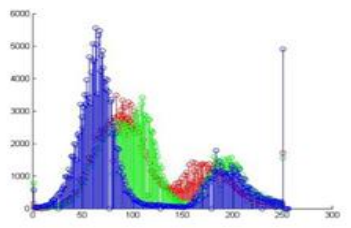

EIAMSRCRIE Output
Histogram Equalisation

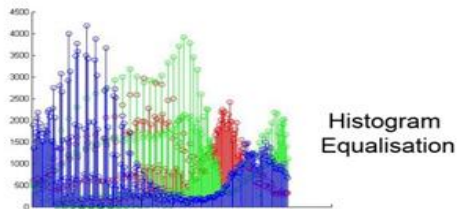

Fig 3. Histograms of Sample Image 1 and the Enhanced Image

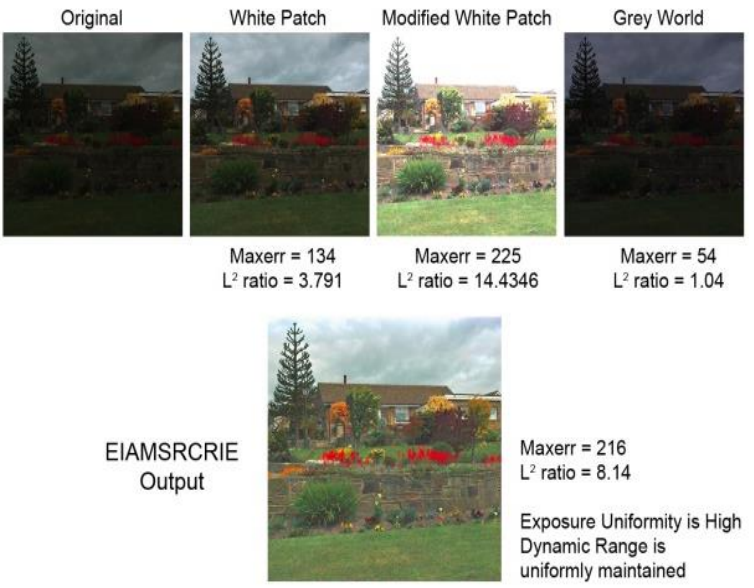

Fig 4. Comparative Analysis of this method with other available methods

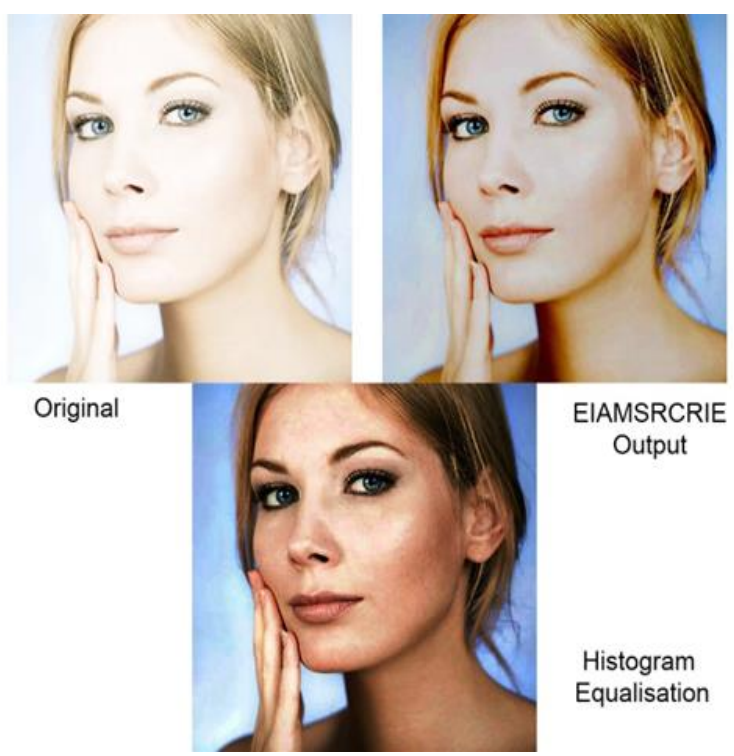

Fig 5. Sample Image 2 (High Key) and the Enhanced Image
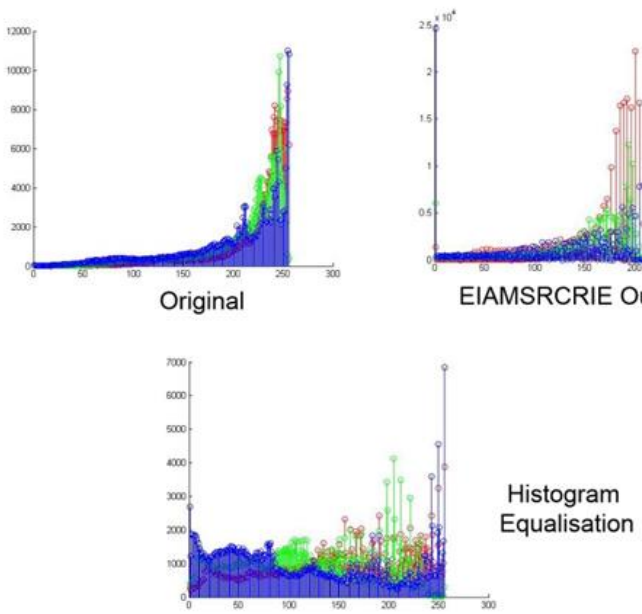

Fig 6. Histograms of Sample Image 1 and the Enhanced Image

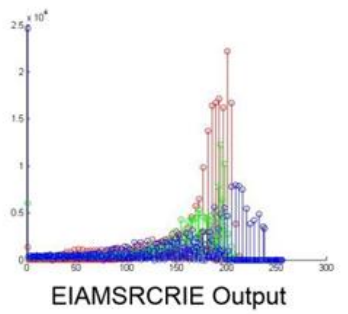
Sciences Publication 
Above figures show a sample of a low key image and a high key image 512x512. The comparative results of this procedure with other procedures are also shown. The maxerr is chosen as the comparative parameter to show the maximum squared deviation of values between the original and the enhanced image. The L2ratio parameter was also selected as the comparative parameter to show squared norm of the image approximation.

From the histogram one can see that the histogram centering is achieved along with preservation of variations in the intensity values. The Maxerr and L2rat values of the comparative analysis show the enhancement factor that has improved the image and also no perceiver can deny the fact that the image enhanced through this procedure is better than all the compared images.

From the results the strong variations in the luminance values among the different areas in the image is suppressed giving a uniformly lit look. This look is vital for any photograph unless there is a play of lighting situations involved in the scene. Due to this property the dynamic range of the image captured is also greatly increased regardless of the sensitivity of the capturing sensor used. The effective and efficient handling of the high key images is also an added advantage to the procedure. The details that it brings out from the high key images or low key images are extraordinary. Even if a perfect image shot under favourable lighting conditions is passed through this algorithm the result is the same image with little more details getting added to it. The algorithm was passed on various test images ranging over two hundred but, for all the images the results were great.

\section{CONCLUSION}

During the research period of the project the need for a system which automatically enhances images and continuous frames was wide. In live location shoots one can never be certain that the lighting conditions may always be favourable to the subject. In order to avoid certain type of degradations to images and to give the perceiver a better look of the scene and also to make him feel himself as a person in the scene this algorithm was developed. It has its scope in almost all areas involving an image sensing device to capture a scene.

The next propagation of this project will be a hardware realization involving real-time processing of continuous frames. With the availability of multi core GPU processing units this task can be easily achieved through efficient transfer of this algorithm to hardware. The project will surely revolutionize the media world reducing the distance between the perceiver and the location of the scene.

\section{REFERENCES}

1. Parthasarathy, S.; Sankaran, P., "An automated Multi Scale Retinex with Color Restoration for image enhancement," Communications (NCC), 2012 National Conference on, vol., no., pp.1,5, 3-5 Feb. 2012 doi: 10.1109/NCC.2012.6176791

2. R. C. Gonzalez, R. E. Woods, "Digital Image Processing," Third Edition, Pearson Publications.

3. S. M. Pizer, E. P. Amburn, "Adaptive histogram equalization and its variations," Computer Vision, Graphics and Image Processing, vol. 39, pp. 355-368, 1987.

4. E. Provenzi, C. Gatta, M. Fierro and A. Rizzi, "A Spatially Variant White-Patch and Gray-World Method for Color Image Enhancement Driven by Local Contrast," in IEEE Transactions on Pattern Analysis and Machine Intelligence, vol. 30, no. 10, pp. 1757-1770, Oct. 2008 doi: 10.1109/TPAMI.2007.70827

5. Zia-ur Rahman, Daniel J. Jobson, Glenn A. Woodell "Retinex processing for automatic image enhancement", J. Electron. Imaging. 13(1), 100-110 (Jan 01, 2004)

6. W. J. Kyung, Y. H. Ha, D. C. Kim, "Real time Multi Scale Retinex to enhance night scene of vehicular camera," 17th Korea-Japan Joint Workshop on Frontiers of Computer Vision, 2011.

7. Cha, Hyo-Sang, Hong, Sung-Hoon, “Advanced Retinex Algorithm for Image Enhancement" Volume 16, Issue ,1, 2013, Journal of Korea Multimedia Society.

8. M.C. Su, J.H. Guo, D.T. Lin, and G.C. Wang, "New Compensation Algorithm for Color Backlight Images," Neural Networks 2002, IJCNN '02. Proc. of the 2002 International Joint Conference, Vol. 2, pp. 1396-1400, 2002.।

9. Woodell G, Jobson D, Rahman Z (2005) Enhancement of imagery in poor visibility conditions. In: SPIE, sensors, and command, control, communications, and intelligence technologies for homeland security and homeland defense, vol 5778, pp 1-11, 2005.

10. Joshi, K. R.; Kamathe, R. S., "Quantification of retinex in enhancement of weather degraded images," Audio, Language and Image Processing, 2008. ICALIP 2008. International Conference on , vol., no. pp.1229,1233, 7-9 July 2008.

11. Lee, Woo-Ram, Dong-Guk Hwang, and Byoung-Min Jun. "Recovering Color of Dark Images Using Tone Mapping and MSRCR." (2014). International Journal of Software Engineering and Its Applications Vol.8, No.1 (2014)

\section{AUTHORS PROFILE}

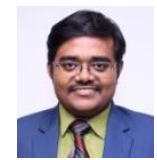

Immanuel K., completed Bachelor of Engineering, Electronics and Communication Engineering at St. Joseph's College of Engineering, Anna University, Chennai, India and Masters of Engineering, Applied Electronics in College of Engineering Guindy, Anna University, Chennai, India. He is currently working as Assistant Professor, in Department of Electronics and Communication Engineering, St. Joseph's Institute of Technology, OMR, Chennai. He is having a teaching experience of 5 Years. He has keen research interest in areas of Image Processing, Machine Learning, Deep Learning and IOT.

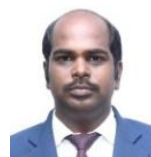

Dinesh Kumar A., completed Bachelor of Engineering, Electronics and Communication Engineering at Institute of Road and Transport Technology, Anna University, Chennai, India and Masters of Engineering, Applied Electronics in Sri Krishna Engineering College, Anna University, Chennai, India. He is currently working as Assistant Professor, in Department of Electronics and Communication Engineering, St. Joseph's Institute of Technology, OMR, Chennai. He is having a teaching experience of 4 Years. He has keen research interest in areas of Signal Processing, Antenna Design, IOT and Embedded Systems.

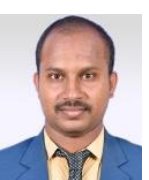

Praveen Kumar E., completed Bachelor of Engineering, Electronics and Communication Engineering at Hindusthan Institute of Technology, Anna University, Coimbatore, India and Masters of Engineering, Communication Systems in Bannari Amman Institute of Technology, Erode, Sathyamangalam India. He is currently working as Assistant Professor, in Department of Electronics and Communication Engineering, St. Joseph's Institute of Technology, OMR, Chennai. He is having a teaching experience of 5 Years. He has keen research interest in areas of Image Processing, IOT and Wireless Communications. 